\title{
Evaluation of Quantitative Sulfur Speciation in Gas Oils by Fourier Transform Ion Cyclotron Resonance Mass Spectrometry: Validation by Comprehensive Two-Dimensional Gas Chromatography
}

\author{
Hendrik Muller, Frederick M. Adam, Saroj K. Panda, Hanadi H. Al-Jawad, Adnan A. Al-Hajji \\ Research and Development Center, Saudi Aramco, P.O. Box 62, Dhahran 31311, Saudi Arabia
}

\begin{abstract}
Fourier transform ion cyclotron resonance mass spectrometry (FT-ICR MS) has been applied for the quantitative speciation of sulfur containing compounds in gas oil (GO). For this purpose, ionization and mass spectrometric parameters have been studied and optimized with a set of standard compounds and GO samples. Comprehensive two-dimensional gas chromatography (GCxGC) was used as the reference method. To allow a quantitative comparison between FT-ICR MS and GCxGC results for GO samples, FT-ICR MS parameters were optimized and data obtained by both techniques were standardized. Response factors were established for two ionization modes: atmospheric pressure photo ionization (APPI) and electrospray after selective derivatization of sulfur compounds (MeESI). To test the validity of the developed MS methods, a third GO was analyzed and response factors were applied. Comparison with GCxGC results showed good agreement for sulfur families (deviation within $5 \%$ and $15 \%$ for MeESI and APPI data, respectively). Abundances of individual isomer groups match within $40 \%$ in most cases. These results principally demonstrate the suitability of FT-ICR MS for a quantitative analysis of sulfur compounds (by DBE and carbon number distribution pattern) in petroleum middle distillates. This approach has the potential to be extended to higher- and non-boiling petroleum fractions where quantitative speciation is presently not available.
\end{abstract}

Key words: Atmospheric pressure photo ionization, Quantitation, Fourier transform ion cyclotron resonance mass spectrometry, Organic sulfur compounds, Petroleum, Gas oil, Comprehensive two-dimensional gas chromatography

\section{Introduction}

$\mathrm{T}$ he rising demand of transportation fuels in combination with changing feedstock supplies lead refiners to process heavier and sourer crude oils. An integral part of today's

Electronic supplementary material The online version of this article (doi:10.1007/s13361-011-0321-7) contains supplementary material, which is available to authorized users.

Correspondence to: Hendrik Muller; e-mail: hendrik.muller@aramco.com refining process is the reduction of sulfur levels to meet product specifications. For example, new processes for deep desulfurization are sought for the cost-effective production of clean transportation fuels out of increasingly challenging feedstocks. Process development, comparison of processes, and selection of feedstocks all require in-depth characterization of heteroatom containing compounds, particularly sulfur-containing molecules, in feed and product streams [1, 2]. However, a quantitative characterization at the molecular level is only available for light and middle distillates, difficult for higher boiling cuts, and not available for non-boiling fractions [3].

Received: 27 June 2011

Revised: 12 December 2011

Accepted: 14 December 2011

Published online: 23 February 2012 
Gas chromatography (GC) and comprehensive twodimensional GC (GCxGC) have been used extensively to provide quantitative information on low- and mid-boiling distillation cuts [4], but are inherently limited by analyte boiling point to a maximum of $400{ }^{\circ} \mathrm{C}$ to $500{ }^{\circ} \mathrm{C}$ [5-8]. In addition, the complexity of hydrocarbon mixtures increases rapidly with the boiling point, thereby making a complete separation and identification of all components by chromatographic means difficult [9]. Because of the limits of chromatographic methods, higher boiling fractions [10] and vacuum residues (heavy ends) [11], bitumen [12], and whole crude oils [13] have been routinely characterized qualitatively by Fourier transform ion cyclotron resonance mass spectrometry (FT-ICR MS); however, quantitative FT-ICR MS applications are limited so far [14]. The quantitation of polar compounds in complex mixtures (petroleum vacuum residue and polyethylene glycol 600) by electrospray ionization (ESI) FT-ICR MS was evaluated [15] and principally deemed possible. A linear response up to reasonable concentrations was achieved after the optimization of the external ion accumulation period. The potential use of ESI FT-ICR MS as a semiquantitative tool to monitor the production of naphthenic acids during crude oil biotransformation was also investigated [16], and the quantitation of ARN-type acids in whole crude oils has been reported [17].

FT-ICR MS in combination with various ionization methods such as ESI, matrix assisted laser desorption ionization, atmospheric pressure photo ionization (APPI) and atmospheric pressure laser ionization was used for a qualitative speciation of sulfur containing compounds in petroleum samples [9]. For the same purpose, a selective derivatization of the nonpolar sulfur species to methylsulfonium salts prior to their analysis was reported [11] but found to exclude molecules with more than six or seven aromatic rings [18]. In this context, APPI was proposed more suitable for the analysis of compounds with higher aromaticity. However, ESI after methylation still provides a valuable alternative means of ionization for mono- to hexa-aromatic sulfur compounds regardless of their boiling point, particularly in fractions not amenable to alternative analytical methods. APPI in conjunction with FT-ICR MS has been widely used for the characterization of sulfur species [19, 20] because of its applicability to non-polar aromatic compounds. The role of the solvent as dopant for proton transfer in APPI has been investigated [21, 22]. The formation of protonated molecules in case of petroleum samples was attributed to proton transfer from components of the petroleum matrix rather than from the dopant [23].

To our knowledge, the quantitation of sulfur containing compounds in petroleum using FT-ICR MS has not been reported to date. In this work, we assessed FT-ICR MS with different ionization modes for a quantitative characterization of sulfur aromatic compounds. The focus of this study lies on gas oil (GO) samples because these can be quantitatively speciated using GCxGC and, therefore, used as a reference. The desired comprehensive quantitative analysis of aromatic sulfur species by FT-ICR MS carries a potential of being readily extended to higher boiling fractions, where quantitative speciation is not available today.

\section{Experimental}

\section{Sample Selection}

For this study, three straight run GO samples with similar boiling ranges from $150{ }^{\circ} \mathrm{C}$ to $395{ }^{\circ} \mathrm{C}$ were selected. The samples are listed in Table 1. GO1 and GO2 were distilled from two different Arabian crude oils and have high total sulfur content. GO3 was distilled from an Asian crude oil and has approximately half the sulfur content of GO1 or GO2.

A standard mixture of four sulfur compounds was prepared to study the effect of the number of aromatic rings within a molecule on its ionization efficiency. The mixture was prepared from four individual sulfur standards obtained from Chiron AS, Trondheim, Norway: diphenylsulfide, $3 n-$ heptylthiophene, trimethylbenzothiophene (46:54 mixture of 2,3,4 and 2,3,6), and dibenzothiophene, each dissolved at approximately $1000 \mathrm{ppm}$ in isooctane. The actual concentration ratio of the mixture was determined by $\mathrm{GC}$ with flame ionization detector (FID) and confirmed with GCsulfur chemiluminescence detector (SCD). This stock mixture was diluted: (1) 1:100 in dichloromethane (DCM), Fisher Scientific, GLC pesticide grade, and (2) toluene (TOL), Riedel de Haen, extra pure, before analysis by APPI FT-ICR MS. Second, the stock mixture was also derivatized by selective methylation [11] of the sulfur atoms before analysis by ESI FT-ICR MS. In this case, the sulfur compounds exist already in ionic form in solution before infusion by a conventional ESI source. We refer to this process as methylation electrospray (MeESI) to distinguish the different mechanism from standard ESI.

\section{Methylation of Sulfur Compounds}

Derivatization of the sulfur standards and GO samples with methyl iodide was carried out according to a reported procedure [11] to provide an alternative ionization mode to APPI. Unless stated otherwise, chemicals were purchased from Sigma Aldrich, Germany. About $50 \mathrm{mg}$ of each sample was treated with an excess amount of methyl iodide (purum) and silver tetrafluoroborate (98\%) in 1,2-dichloroethane for $48 \mathrm{~h}$. The obtained silver iodide precipitate was removed by filtration and washed with $2 \mathrm{~mL}$ of 1,2-dichloroethane. The filtrates containing the methylsulfonium salts were further

Table 1. Geographical Origin and Total Sulfur Content of Straight Run GO Samples

\begin{tabular}{lcc}
\hline Code & Geographical origin & Sulfur $[\%(w t)]$ \\
\hline GO1 & Middle East & $1.30 \%$ \\
GO2 & Middle East & $1.23 \%$ \\
GO3 & Asia & $0.69 \%$ \\
\hline
\end{tabular}


diluted 1:1,000 in DCM or acetonitrile (ACN) (chromasolve) before their characterization by positive mode ESI FT-ICR MS.

\section{Mass Spectrometry}

Mass spectra were acquired using a 9.4 Tesla Apex Qe Fourier transform ion cyclotron resonance mass spectrometer (Bruker Daltonics, Bremen, Germany) with two ionization modes. An Apollo II ion source, operated in the positive ESI mode, was used for external mass calibration and infusion of methylated samples [20]. Additionally, the Apollo II ion source in the positive APPI mode was used for photoionization. A wide variety of ionization and mass spectrometric parameters were tested. Here we provide the optimized values; the influence of critical parameters is discussed in the text.

\section{Ionization}

For each GO sample, ionization was done in different solvents and ionization modes. Using APPI, the samples were diluted in TOL, and in a separate experiment, in DCM. Both solutions were diluted (1:1,000 vol/vol) and delivered by syringe pump at flow rate of $1.2 \mathrm{~mL} / \mathrm{h}$. In a second set of experiments, all samples were ionized using methylation ESI after dilution in acetonitrile and, in a second set of experiments in dichloromethane. All solutions were diluted $(1: 1,000 \mathrm{vol} / \mathrm{vol})$ and delivered by syringe pump at $0.24 \mathrm{~mL} / \mathrm{h}$. Gas flow rates (nebulizer and dry gas) were set at $3 \mathrm{~L} / \mathrm{min}$. APPI furnace temperature was set at $300{ }^{\circ} \mathrm{C}$. Ion source potentials were set as follows: Capillary: 1.5 and $4.5 \mathrm{kV}$, spray shield: 1.0 and $4.0 \mathrm{kV}$ for APPI and MeESI, respectively, capillary exit: $270 \mathrm{~V}$.

\section{Mass Spectrometer}

Source and quadrupole accumulation times were set to $0.1 \mathrm{~s}$ each, ion transfer time (TOF) was optimized to $0.8 \mathrm{~ms}$. A modification of analyzer settings (excitation amplitude, trapping plate potentials and sidekick voltage) did not have adverse effects on results as long as unperturbed mass spectra with symmetric mass signals were obtained. For all measurements, masses were recorded from 115 to $3000 \mathrm{~m} / \mathrm{z}$; 128 raw spectra were co-added before fast Fourier transformation to reach a compromise between signal to noise ratio and measurement time. Although beyond the scope of this work, it seems advisable for future work to examine ICR excitation and detection specifically with quantitation aspects in mind.

\section{Mass Calibration}

External mass calibration was conducted on a daily basis using sodium formate solution ( $5 \mathrm{mMolar}$ in water/methanol
1:1) in positive mode ESI. Internal calibration for each sample measurement was based on homolog series of benzothiophenes (BTs) and/or aromatic hydrocarbons present in the samples. Data were acquired on ApexControl software (ver. 2.0, Bruker Daltonics, Bremen, Germany).

\section{Elemental Composition Assignment}

Elemental compositions $\left(\mathrm{C}_{\mathrm{c}} \mathrm{H}_{\mathrm{h}} \mathrm{N}_{\mathrm{n}} \mathrm{O}_{\mathrm{o}} \mathrm{S}_{\mathrm{s}}\right)$ were calculated for all mass signals using Composer software (ver. 1.0.1, SierraAnalytics, Modesto, CA, USA) and in-house developed Excel (Microsoft, Redmond, USA) visual basic macros. Elemental composition assignments were confirmed by the ${ }^{34}$ sulfur isotopic fine structure for sulfur series signals $[20,24]$. In case of methylated samples, the methyl group was subtracted from elemental compositions of sulfur species. The identified elemental compositions were then classified according to the number and type of heteroatoms (e.g., hydrocarbons, mono-sulfur $\left(\mathrm{S}_{1}\right)$, or mono-nitrogen species for molecules with one sulfur or nitrogen atom). The corresponding double bond equivalent (DBE) values and carbon numbers were calculated for each identified elemental composition. The DBE is a measure of the aromaticity of a molecule, and it is defined as half the number of hydrogen atoms lacking from the completely saturated molecule with otherwise identical number of carbon and heteroatoms. Unless stated otherwise in the text, the protonated or methylated ion species and their respective isotope peaks ( ${ }^{13}$ carbon and ${ }^{34}$ sulfur) were summed and used for quantitation purposes by APPI and MeESI.

\section{Gas Chromatography}

Two-dimensional comprehensive gas chromatograms were obtained using a 7890 Agilent GC (Agilent Technologies, Santa Clara, CA, USA) modified with a single loop modulation system from Zoex (ZX1 model; Zoex Corporation, Houston, TX, USA). The modulation period was set at $12 \mathrm{~s}$. For this work the GCxGC was equipped with a SCD from Agilent (Santa Clara, California, CA), which was operated as recommended by the manufacturer. System performance (detection limit, linearity, and equimolarity) was systematically assessed before analysis. Samples $(0.5$ $\mu \mathrm{L})$ were injected using a cool on-column injector. The separation was carried out at a constant flow rate of $1.3 \mathrm{~mL} /$ min helium as carrier gas. The first column had a polydimethylsiloxane stationary phase (DB1, $15 \mathrm{~m}$, $0.25 \mathrm{~mm}, 250 \mu \mathrm{m})$ and the second column $50 \%$ phenylsiloxane $/ 50 \%$ polydimemthylsioloxane (BPX $50,1.2 \mathrm{~m}$, $0.1 \mathrm{~mm}, 100 \mu \mathrm{m})$. The oven temperature was ramped from $50{ }^{\circ} \mathrm{C}$ to $300{ }^{\circ} \mathrm{C}$ at a rate of $2{ }^{\circ} \mathrm{C} / \mathrm{min}$.

Data processing was achieved using image software (Zoex Corporation, Houston, TX, USA). The chromatographic identification of compounds was based on the retention times of standard compounds and literature [25, 
26]. Quantification was achieved by normalizing the chromatogram to the total sulfur content [25].

\section{Results and Discussion}

The capability of FT-ICR MS for the quantitative analysis of sulfur aromatic compounds was studied because this approach, unlike gas chromatographic methods, can be extended to petroleum heavy ends. The basic characteristics of FT-ICR MS for quantitation were investigated using standard compounds. Then, because sulfur compounds in GO are amenable to GCxGC and, therefore, can be quantitatively determined as a reference, GO samples were used to optimize FT-ICR MS parameters and for demonstrating the quantitative relevance of the FT-ICR MS data.

\section{Quantitation Characteristics}

Basic quantitative performance characteristics, including the dynamic range, linearity, robustness, and ruggedness of the APPI FT-ICR MS were initially assessed using standard solutions (1.4 to $2301 \mathrm{ppm}$ ) of dibenzothiophene in toluene. The signal response was found to be slightly nonlinear $\left(y=2^{7 *} x^{0.6753}, R^{2}=0.999\right.$, see Supplementary Information Figure S1). The dynamic range (minimum detectable signal intensity in relation to the most intensive signal) covers at best three orders of magnitude. Assuming comparable ionization efficiency between analytes and petroleum matrix, the limited dynamic range restricts the minimum detectable level of sulfur aromatic compounds to $0.1 \%-1 \%$ of the most abundant aromatic hydrocarbon present in the sample [27]. Therefore, only samples with significant sulfur content can be analyzed if a non-selective ionization is employed. The limit of detection was not determined, because FT-ICR MS measurements can be easily adapted to lower concentrations (sub ppm level) by: (1) averaging a greater number of raw spectra, (2) increasing the ion accumulation time, and (3) sending more than one ion package into the ICR cell for each measurement. The repeatability over 10 successive measurements yielded a standard deviation below $11 \%$. The ruggedness for an absolute response measurement after a complete shutdown and restart of the vacuum system was poor; however, the ruggedness for relative response (i.e., patterns of sulfur compounds) after 6 mo and after 2 y was good (within $11 \%$ standard deviation per signal).

For quantitative purposes a response curve that is independent of molecular features of the analyte is desirable; however, information on the ionization behavior of sulfur aromatic compounds in APPI and MeESI is scarce. To establish the response behavior of aromatic sulfur compounds in MS analysis systematically, the impact of molecular structure on ionization efficiency was studied from two angles: (1) the effect of DBE of a molecule, and (2) the impact of the number of carbon atoms within a given DBE series. The effect of DBE on the ionization efficiency was studied first using a mixture of sulfur standards, and then GO samples using quantitative GCxGC-SCD analytical results as reference. The response depending on carbon numbers within a given DBE series was then investigated for GO samples in comparison to speciation data by GCxGC.

\section{Ionization of Standards}

A mixture of four sulfur compounds that are expected in GO was analyzed using APPI and MeESI to establish a basic understanding of their ionization behavior. To minimize ion transfer phenomena that are suspected of discriminating based on molecular weight, standards with similar masses but of different molecular structures for the sulfur atom were selected (sulfide, thiophene, benzothiophene, and dibenzothiophene). The mixture was diluted $(1: 100 \mathrm{vol} / \mathrm{vol})$ in TOL to a concentration of $2 \mathrm{ppm}$ for each compound. A second solution $(1: 100 \mathrm{vol} / \mathrm{vol})$ was prepared in DCM. In APPI mode, radical ions and protonated molecules were observed for both solvents, except for thiophene, which produced only protonated species (Figure 1a). Ionization yields obtained
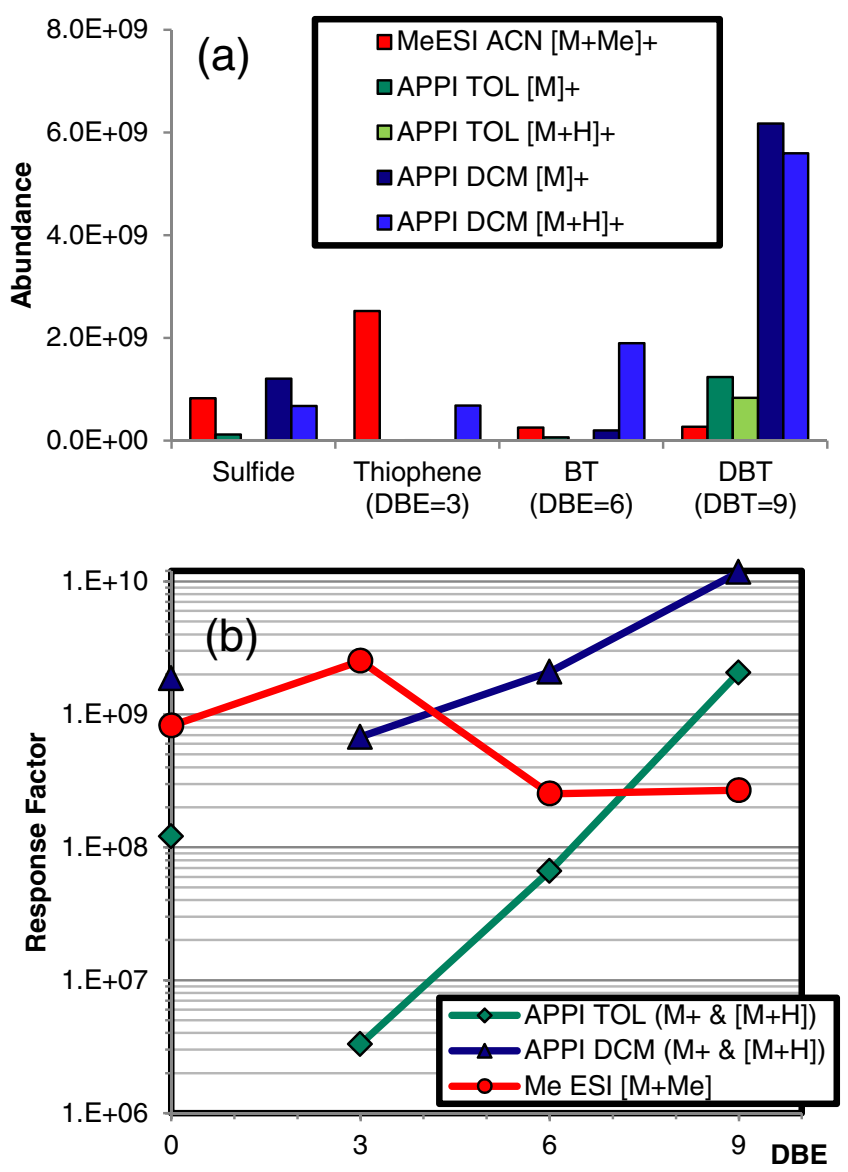

Figure 1. (a) Ion abundances for four sulfur standards with APPI toluene (TOL) and dichloromethane (DCM) and ESI after selective methylation of sulfur compounds (sulfide, thiophene, benzothiophene, and dibenzothiophene). Ion abundances have been normalized to represent an equimolar mixture of $2 \mathrm{ppm}$ per standard. APPI yielded molecular ions and protonated molecules, the ESI spectra showed only methylated ions. (b) Response factors of combined $\mathrm{M}^{+}$and $[\mathrm{M}+\mathrm{H}]^{+}$species versus DBE value of standard molecules on a logarithmic scale 
from a DCM solution were higher than those from a TOL solution. An influence of the molecular structure, particularly DBE values, was found. As expected, analytes with higher DBE value ionize more efficiently in APPI mode. The standard mixture was also methylated and infused by ESI mode. Using DCM as a solvent, signals for radical ions, protonated and methylated molecules were observed. In contrast, dilution in ACN produced exclusively methylated molecules. Abundances of methylated ions were not apparently related to the DBE values of the molecules (red bars in Figure 1a).

To compare the APPI response factors of the standards the abundances of radical ions and protonated molecules were summed and plotted against the DBE values (Figure 1b). A DCM solution yielded approximately two orders of magnitude higher response factors compared to a TOL solution at equal concentration (blue and green line in Figure 1b, respectively). For both solvents, the response factors increased with DBE, with the exception of diphenylsulfide, because the two phenyl groups increase the ion yield unduly in APPI mode (the sulfur atom is not part of the aromatic system; therefore, the sulfide is shown at $\mathrm{DBE}=0$ in Figure 1b). For a DCM solution, the response factors increase from thiophene to DBT about one order of magnitude. The TOL solution showed an even higher response dependency regarding the DBE value with an increase over two orders of magnitude from thiophene to DBT. MeESI after dilution (1:1000) in acetonitrile yielded only methylated species $\left([\mathrm{M}+\mathrm{Me}]^{+}\right)$with response factors varying over one order of magnitude.

The ionization of sulfur aromatic compounds by APPI and MeESI was also explored for GO samples, where the exact concentrations were unknown. To this end, characterization results by GCxGC were obtained and used as a reference for the FT-ICR MS data.

\section{Gas Oils by GCxGC}

Sulfur species were separated according to their boiling points and polarities (related to the degree of aromaticity of a molecule) on the first and the second column, respectively. The separation resulted in a two-dimensional (2D) chromatogram as shown in Figure 2a, which was obtained for GO1. In the chromatogram the main series (circled signals) of thiophenes (Ts), BTs, dibenzothiophenes (DBTs), and benzonaphthothiophenes (BNTs) are separated from each other according to their aromaticity along the y-axis of the chromatogram. Simultaneously, molecules with one or two saturated rings (naphtheno-substituted BTs and DBTs) are partially separated along the $y$-axis and are found above their parent structures in the chromatogram. Molecules with different numbers of carbon atoms (in alkyl chains) are separated along the $\mathrm{x}$-axes. In addition, isomers can be separated within each series [28] [e.g., several dibenzothiophenes with 1 alkyl carbon atom $\left(\mathrm{C}_{1}\right.$-DBT) $]$. Due to the lack of chromatographic resolution for highly alkylated sulfur compounds like $\mathrm{C}_{11+}-\mathrm{BT}$ and $\mathrm{C}_{7+} \mathrm{DBT}$, these compounds are routinely quantified as lumped groups. The corresponding elution zones are indicated as shaded areas in Figure 2a. Whereas a series refers to a set of molecules with a fixed aromatic system and a variable number of carbon atoms in alkyl groups (but without naphthenic rings), families are here defined to include all molecules of one series in addition to the molecules substituted with naphthenic rings. The families were found in Figure 2a between the black lines (BT family, DBT family, etc.).

\section{Data Standardization}

Accurate mass determination alone does not allow for the differentiation between isomers which can be at least partially resolved by GCxGC (Figure 2a). To standardize the GCxGC data format to that of the FT-ICR MS, the intensities of the isomer groups obtained by GCxGC were summed to yield one data point for each group. In this format, the GCxGC data resembles the mass spectral data (DBE versus carbon number versus intensity) as shown in Figure $2 b$ [29]. In this graph, data points at higher DBE values represent structures with a higher degree of unsaturation, while dots at higher carbon numbers represent larger molecular sizes. The area of each data point represents the relative intensity of the associated isomer lump obtained from the GCxGC data. The main DBE series of sulfur compounds, namely BTs $(\mathrm{DBE}=6)$ and $\mathrm{DBTs}(\mathrm{DBE}=9)$, can be seen relatively more abundant than the corresponding series with naphthenic rings (Figure 2b). A distinction between structures with or without naphthenic rings cannot be justifiably determined from the GCxGC data (Figure 2a). Therefore, abundances for the families of sulfur compounds were obtained by summing the respective signals, e.g., molecules with and without naphthenic rings like BTs $(\mathrm{DBE}=6)$ and naphtheno BTs (DBE 7 and 8 ) into one family that includes DBE 6-8).

\section{Gas Oils by FT-ICR MS}

Aromatic compounds, including sulfur containing molecules, were ionized without fragmentation. Masses were measured with sufficient resolution and accuracy to identify underlying elemental compositions. Elemental compositions with one sulfur atom (S1) were the only sulfur containing class found in GO1 and GO3. The carbon number versus DBE plots for S1 species for MeESI, and APPI using DCM and TOL, are provided in Figure 3. In contrast to the GCxGC, the mass spectral data allows for a clear distinction between all DBE series. A variation of abundances in dependence of DBE could be observed between the different ionization modes. MeESI data (Figure 3a) shows abundant species at lower DBE values (DBE 1 to 5), regardless of the used solvent. APPI data shows molecules with higher DBE values (DBE 9 to 11), shown in Figure $3 \mathrm{~b}$ and c, particularly when TOL is used as a solvent (Figure 3c). Interestingly, few S2 compounds were found in $\mathrm{GO} 2$ after selective methylation; one series of S2 class compounds with DBE 

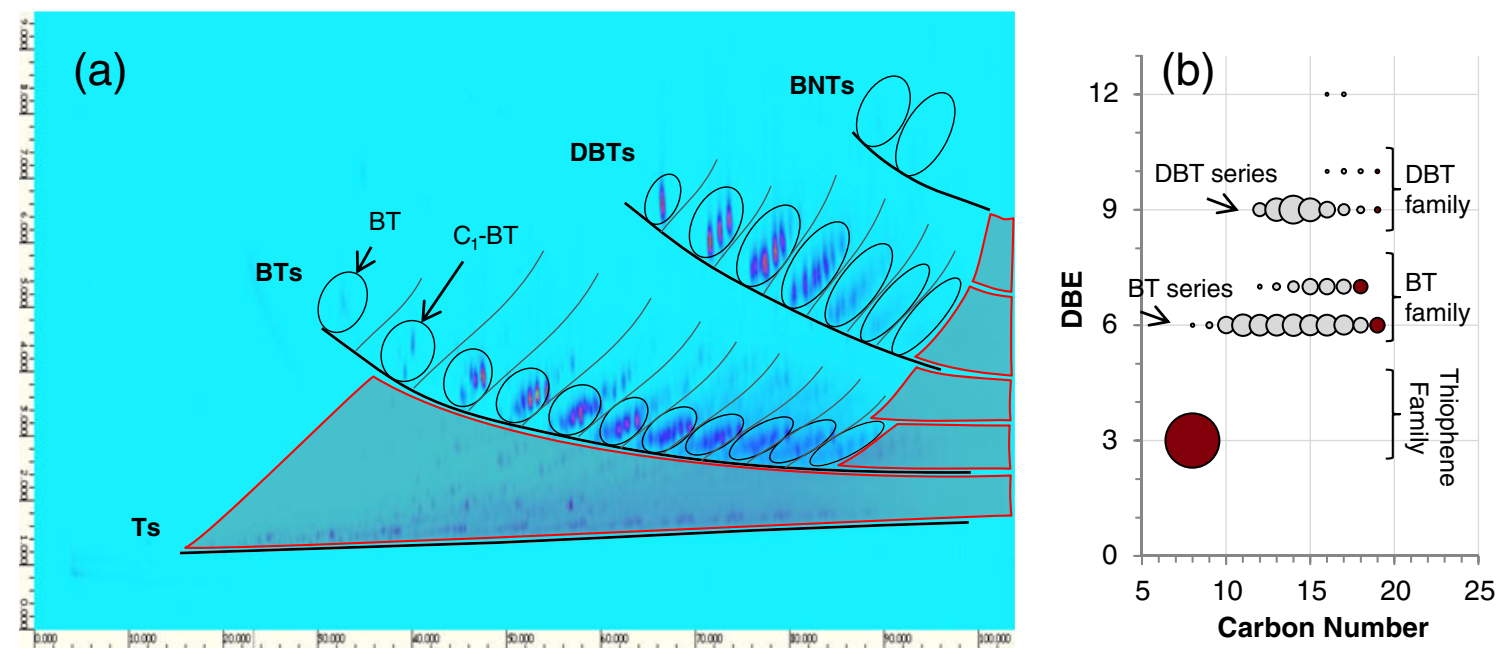

Figure 2. (a) Two-dimensional sulfur selective gas chromatogram of G01. Main series are marked by ovals: Thiophenes (Ts), benzothiophenes (BTs), dibenzothiophenes (DBTs), and benzonaphthothiophenes (BNTs). Areas between the main series contain naphtheno and dinaphtheno substitutes of the parent series below and are lumped together as one compound family. Shaded areas correspond to lumped signal groups. (b) Double bond equivalent versus carbon number breakdown of standardized GCxGC data. The area of the dots represents summed intensities for each respective isomer group. The red dots represent lumped signals in incompletely resolved areas

8 and carbon numbers 13 to 17 was detected with low relative abundance $(0.1 \%$ summed ion abundance). DBE 8 could correspond to benzothienothiophenes (or isomers thereof) with additional 3 to 7 carbon atoms as alkyl groups, which are plausible sulfur containing structures that could be indigenous to petroleum samples. Because of their low abundance in the studied GO samples, S2 molecules were not considered for this study. However, it should be noted that S2 species are expected to be more abundant in higher boiling fractions and, therefore, should be considered for future studies.

\section{Carbon Number Distributions}

An APPI mass spectrum obtained for GO1 is provided in the supplementary information (Figure S2) with signal intensity profiles of the BTs and DBTs series highlighted. To find the best TOF, mass spectra were recorded from 0.6 to $2.0 \mathrm{~ms}$ and the relative profiles for the BT and DBT series were compared to the reference profiles obtained by GCxGC. Provided that there is a match between the profiles from the two techniques, a quantitative breakdown per carbon atom numbers could be realized from the mass spectra.

A comparison of the BT and DBT carbon number distributions by APPI FT-ICR MS and GCxGC is provided in the supplementary information (Figure S2). The best match between the two techniques is found at $0.8 \mathrm{~ms}$ TOF in case of the studied GO samples. Results for GO2 were similar to that of GO1 and, therefore, confirm this conclusion. GCxGC and FT-ICR MS normalized distributions profiles for $\mathrm{C}_{0}$ to $\mathrm{C}_{7}$ alkyl substituted DBTs (carbon
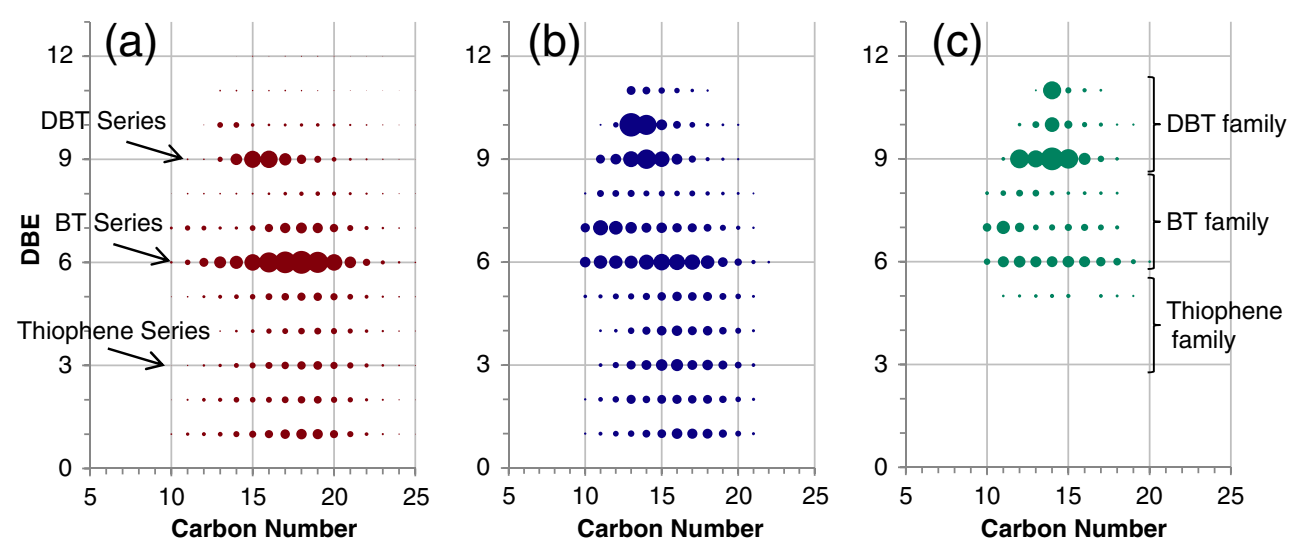

Figure 3. (a) Double bond equivalent versus carbon number plots of the S1-class compounds detected by MeESI in ACN. Methyl groups introduced by the derivatization have been subtracted. The size of the dots represents the summed abundance for each isomer group. (b) S1-class species $\left([\mathrm{M}+\mathrm{H}]^{+}\right)$detected using APPI with DCM as solvent. (c) S1-class species $\left(\mathrm{M}^{+}\right)$ measured using APPI with TOL as solvent 
numbers 12 to 19) are similar, with the exception of DBT (carbon number 12) which is determined significantly higher by MS than by GCxGC. For the BT family, similar agreement between techniques is observed. However, GCxGC analysis did not resolve $\mathrm{C}_{12+}$-BTs (carbon numbers 20 and higher), which can be resolved by MS. Generally, longer ion transfer time results in an overestimation of species with higher molecular weight. Using MeESI and setting $\mathrm{TOF}=0.8 \mathrm{~ms}$, similar carbon number distributions were obtained for GO1 and 2 (not shown). The observed impact of ion transfer time on carbon number distributions, even for GO samples with a comparably narrow molecular weight distribution, should be carefully considered in further FT-ICR MS studies of petroleum.

\section{Concentration Effect}

To evaluate and exclude concentration effects on the obtained carbon number profiles, GO1 and $\mathrm{GO} 2$ were diluted to a series of concentrations from 1:100 to $1: 100,000$ in TOL and DCM (APPI) and DCM and ACN (MeESI). Significant changes in the sulfur family abundance ratios were observed for the most highly concentrated solutions (100 and 500 times dilution) in any combination of GO samples and ionization modes. Results obtained for these concentrated solutions deviated significantly from the GCxGC reference data. Spectra of 5000 and further diluted samples showed reduced signal to noise ratios. Consequently, 1000-fold dilutions were used throughout this study.

\section{Response Factors}

Because a significant impact of the solvent on the ionization efficiency had been observed for standard compounds, different solvents were tried to dilute GO1. Ionization modes were operated as follows: APPI with TOL and DCM and MeESI with ACN and DCM. The impact of the solvent on the ionization efficiency can be evaluated by comparing the summed intensities of sulfur families. The respective summed relative abundances for the families of thiophenes, BTs, DBTs, and BNTs are shown in Figure 4a for GO1. GCxGC results are shown for comparison (red bars in Figure 4a). The error bars indicate the standard deviation of three repetitive measurements. Similar results were obtained for GO2 (not shown). No discernible solvent effect could be observed on the relative abundances of sulfur families for the two studied GO samples. These findings agree with a previous observation that in case of petroleum samples the hydrocarbon matrix reduces the impact of solvent [23].

Response factors per sulfur family were calculated as the ratio of summed relative mass spectrometric abundances to $\mathrm{GCxGC}$ results for GO1. Figure $4 \mathrm{~b}$ and $\mathrm{c}$ show the APPI and MeESI FT-ICR MS response factors obtained for GO1 and GO2. Although the response factors were calculated independently for GO1 (black circles) and GO2 (white squares), a common trend is observed (trend line and
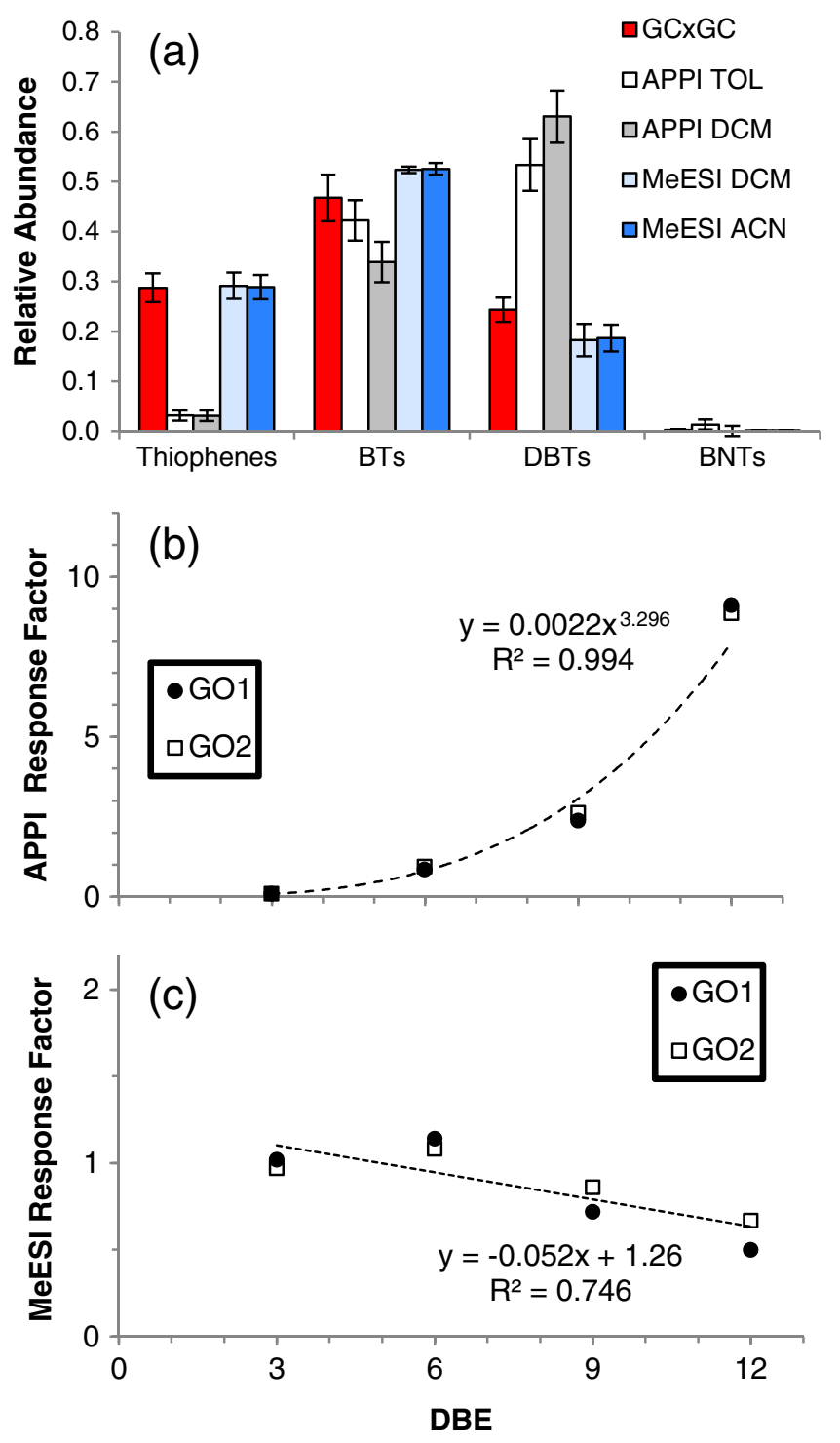

Figure 4. (a) Relative abundances for sulfur families in GO1 by GCxGC (red bars), FT-ICR MS with APPI from TOL (white) and DCM (grey), MeESI from DCM (light blue) and ACN (dark blue). Only $\mathrm{M}^{+}$and $[\mathrm{M}+\mathrm{Me}]^{+}$ion species were considered for APPI and MeESI, respectively. Error bars show the variance for 3 repeated measurements. (b) Response factors versus DBE values by FT-ICR MS APPI (TOL, $\mathrm{M}^{+}$ions) in reference to GCxGC results for GO1 (black circles) and GO2 (white squares). (c) As in (b) but for MeESI, DCM, $[\mathrm{M}+\mathrm{Me}]^{+}$ions

equation are shown in Figure 4b). In APPI mode, response factors show a nonlinear increase with DBE values. This is in agreement with the observations made for sulfur standards (Figure 1b). Likewise, the MeESI response factor curves for $\mathrm{GO} 1$ and GO2 (Figure 4c) are more flat across families, which is similar to the trend observed for standard compounds (Figure 1b). Furthermore, in case of MeESI, a slow decrease of response factors towards higher DBE values can be projected. Extrapolation of the MeESI response factor versus DBE values (equation in Figure 4c) predicts a response factor close to zero (no methylation 
occurring) for $\mathrm{DBE}=24$, which is consistent with previous observations [18].

\section{Quantitative Sulfur Speciation in GO3}

With the observed agreement in terms of carbon number distributions for GO1 and GO2, APPI and MeESI response factors for S1 families were applied for the FT-ICR MS analysis of GO3. Consequently, the total sulfur concentration of GO3 (6850 ppm) was distributed between all individual $\mathrm{S}_{1}$ class species, factored by mass spectrometric abundances and averaged response factors determined before for GO1 and GO2. As a result, individual concentrations can be assigned (in ppm) to each family and for the carbon number breakdowns of main series (Figure 5). The total sulfur distribution per family is shown in Figure 5a. Error bars indicate the uncertainty as determined from three repetitive measurements. It should be noted that in contrast to $\mathrm{GO} 1$ and $\mathrm{GO} 2, \mathrm{GO} 3$ is from a different origin (distilled from an Asian crude oil) and contains significantly less total sulfur. Using APPI for analysis of GO3, only dinaphthenothiophenes $(\mathrm{DBE}=5)$ were detected out of the thiophene family (Figure 5a). A carbon breakdown of the thiophene series, however, could not be provided (Figure 5d) from APPI data.

Using all three methods (APPI, MeESI, and GCxGC), BNTs were found at a negligible level in GO3 (Figure 5a). In comparison to the APPI data (white bars), the MeESI data (grey bars) match closer to the reference GCxGC data (black bars) for all families. After applying the determined response factors, quantitative results for families differ by less than
$15 \%$ between APPI and the reference method and deviate by less than $5 \%$ for MeESI. The determined sulfur family response factors seem applicable for all studied GO samples, regardless of their geographical origins.

The bar graphs in Figure $5 b$ to d show the concentrations (ppm) per carbon number for the DBT, BT and thiophene families in $\mathrm{GO} 3$ as determined by $\mathrm{GCxGC}$, APPI, and MeESI FT-ICR MS. Carbon number distributions obtained by APPI (white bars) match the GCxGC reference distributions (black bars) within $40 \%$, with the exception of DBT (carbon number $(\mathrm{CH})=12$, Figure $5 \mathrm{~b}$ ), $\mathrm{C}_{2}-\mathrm{BT}$ and $\mathrm{C}_{11}$ - $\mathrm{BT}$ $(\mathrm{C} \#=10$ and $\mathrm{C} \#=19$, Figure $5 \mathrm{c}$, respectively). The thiophene series $(\mathrm{DBE}=3)$ was not detected using APPI, instead only naphtheno-thiophenes (DBE $=4$ and 5) were found. $\mathrm{GCxGC}$ did not provide enough separation for the individual quantification of thiophenes and naphtheno thiophenes under the used experimental conditions so that they were lumped together as thiophene family only.

\section{Impact of Ionization Mode}

Ionization process, ion transfer and detection cannot be studied independently for complex samples. Matrix effects might occur at any or all steps of the measurement and a bias of the ionization could be countered by an opposite bias during ion transfer. The fact that two ionization modes achieve representative data increases the confidence that a bias does not occur in the mass spectrometer. In case of standard compounds, APPI is more sensitive towards structures with higher DBE values, whereas MeESI produces a bias towards lower DBE values (Figure 1b). The same
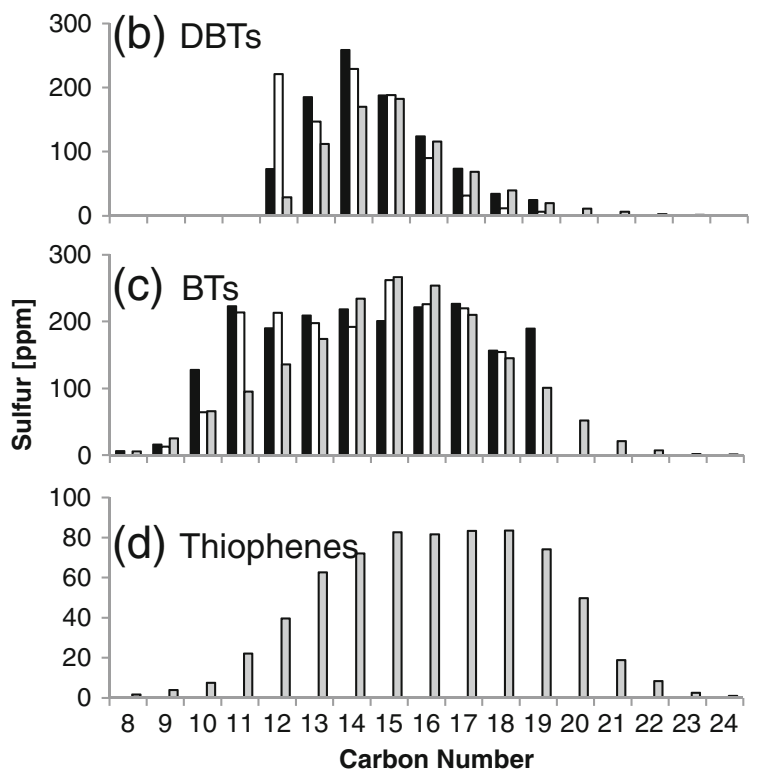

Figure 5. (a) Total sulfur for GO3 S1 Families by GCxGC as a reference (black), FT-ICR MS APPI (white) and FT-ICR MS methylation ESI (grey). (b) Carbon number breakdown for the DBT series in GO3, (c) BT series, and (d) thiophene series; thiophenes $(\mathrm{DBE}=3)$ were ionized only by MeESI and could not be fully resolved by GCxGC. The concentration of sulfur $(\mathrm{ppm})$ in all graphs was obtained by distributing the total sulfur concentration over the identified $\mathrm{S}_{1}$ class families and further between individual carbon numbers, factored by mass signal abundances and response factors determined earlier for GO1 and 2 
trends have been discussed for GO samples (Figure 4a). These biases seem consistent for three GO samples and can be described quantitatively in form of response factor curves. For unknown reasons, the MeESI of GO samples produces carbon number distributions that are approximately one carbon number higher than observed by the reference method, despite the subtraction of one methyl group to compensate for the derivatization. On the other hand, the methylation reaction produces sulfur compound ions more uniformly across DBE values and achieves a more selective ionization than APPI, thereby enhancing less abundant sulfur compound series. For this reason, thiophenes were detected by MeESI but not by APPI. Consequently, the carbon number distribution can be estimated for thiophenes from the MeESI data only (Figure 5d). Even though MeESI seems suitable for the analysis of GO samples the derivatization is limited to sulfur compounds below a DBE value of 24 , preventing the extension of this approach to heavier cuts with more aromatic compounds. The main purpose of this work was to evaluate FT-ICR MS for the ability to yield a quantitative sulfur speciation in a complex hydrocarbon matrix that should be extendable towards higher boiling fractions; therefore, special emphasis was placed on the optimization of APPI parameters and determination of response factors for the main sulfur compound families.

\section{Conclusion}

Targeting a quantitative analysis of sulfur compounds in petroleum samples by FT-ICR MS, ionization and mass spectrometric parameters were tuned iteratively. The ionization by APPI and selective methylation of sulfur compounds followed by ESI were evaluated using sulfur standards and subsequently for two GO samples. Response factors for the main sulfur families were established for both ionization modes; APPI response factors increase with DBE value, whereas methylation ESI response factors decline towards zero at $\mathrm{DBE}=24$. While the ionization of standard compounds is strongly affected by the solvent, this was found not the case for GO samples. For the studied GO samples, the best match between GCxGC and FT-ICR MS data was found at $0.8 \mathrm{~ms}$ ion transfer time (tuned for BT and DBT families). Finally, FT-ICR MS was used for the analysis of a third gas oil sample. Using the established response factors, the concentration of the main sulfur families and carbon numbers for thiophenes, BT, and DBT series were determined. A comparison of FT-ICR MS to a detailed quantitative analysis by $\mathrm{GCxGC}$ as reference method showed a semi-quantitative agreement (between 5\% and $15 \%$ deviation for summed sulfur families and below $40 \%$ deviation for individual carbon number data points). These results demonstrate the capability of FT-ICR MS for obtaining semiquantitative carbon number distributions for the most prominent sulfur-containing compound families in petroleum middle distillates. Further work is already in progress to extend the quantitative speciation of aromatic hydrocarbon and sulfur species by FT-ICR MS towards higher boiling petroleum fractions [27]. A semiquantitative analytical method for vacuum gas oils and vacuum residues, fractions where quantitative speciation is difficult or impossible, is hereby of a particular interest.

\section{Acknowledgment}

The authors acknowledge Dr. Omer R. Koseoglu, Saudi Aramco Research and Development Center, for providing the samples and consultations on the study.

\section{References}

1. Whitehurst, D.D., Isoda, T., Mochida, I.: Present state of the art and future challenges in the hydrodesulfurization of polyaromatic sulfur compounds. Adv. Catal. 42, 345-471 (1998)

2. Stanislaus, A., Marafi, A., Rana, M.S.: Recent advances in the science and technology of ultra low sulfur diesel (ULSD) production. Catal. Today 153, 1-68 (2010)

3. Andersson, J.T., Hegazi, A.H., Roberz, B.: Polycyclic aromatic sulfur heterocycles as information carriers in environmental studies. Anal. Bioanal. Chem. 386, 891-905 (2006)

4. Adam, F.M., Vendeuvre, C., Bertoncini, F.: Comprehensive twodimensional gas chromatography for enhanced analysis of naphthas: New column combination involving permethylated cyclodextrin in the second dimension. J. Chromatogr. A 1178, 171-177 (2008)

5. Altgeld, K.H., Boduszynski, M.M.: Chromatographic separation of heavy petroleum fractions, pp. 203-248. Marcel Dekker, Inc, New York (1994)

6. V Adam, F.M., Bertoncini, F., Brodusch, N., Durand, E., Thiebaut, D., Espinat, D., Hennion, M.C.: New benchmark for basic and neutral nitrogen compounds speciation in middle distillates using comprehensive two-dimensional gas chromatography. J. Chromatogr. A 1148, 5564 (2007)

7. Adam, F., Thiebaut, D., Bertoncini, F., Courtiade, M., Hennion, M.-C.: Supercritical fluid chromatography hyphenated with twin comprehensive two-dimensional gas chromatography for ultimate analysis of middle distillates. J. Chromatogr. A 1086(1-2), 21-28. 1217, 1386-1394 (2010)

8. Adahchour, M., Beens, J., Vreuls, R.J.J., Batenburg, A.M., Brinkman, U.A.T.: Comprehensive two-dimensional gas chromatography of complex samples by using a 'reversed-type' column combination: application to food analysis. J. Chromatogr. A 1086(1-2), 21-28. 1054, 47-55 (2004)

9. Panda, S.K., Andersson, J.T., Schrader, W.: Characterization of supercomplex crude oil mixtures: What is really in there? Angew. Chem. Int. Ed. 48, 1788 (2009)

10. Panda, S.K., Schrader, W., Andersson, J.T.: Fourier transform ion cyclotron resonance mass spectrometry in the speciation of high molecular weight sulfur heterocycles in vacuum gas oils of different boiling ranges. Anal. Bioanal. Chem. 392, 839-848 (2008)

11. Mueller, H., Andersson, J.T., Schrader, W.: Characterization of highmolecular-weight sulfur-containing aromatics in vacuum residues using Fourier transform ion cyclotron resonance mass spectrometry. Anal. Chem. 77, 2536-2543 (2005)

12. Smith, D.F., Schaub, T.M., Rahimi, P., Teclemariam, A., Rodgers, R.P., Marshall, A.G.: Self-association of organic acids in petroleum and Canadian bitumen characterized by low- and high-resolution mass spectrometry. Energy Fuels 21, 1309-1316 (2007)

13. Panda, S.K., Schrader, W., Al-Hajji, A., Andersson, J.T.: Distribution of polycyclic aromatic sulfur heterocycles in three Saudi Arabian crude oils as determined by Fourier transform ion cyclotron resonance mass spectrometry. Energy Fuels 21, 1071-1077 (2007)

14. Shi, Q., Zhao, S.-Q., Xu, C.-M., Hou, D.-J.: Fourier transform ion cyclotron resonance mass spectrometry and its application in petroleum analysis. Zhipu Xuebao 29, 367-378 (2008)

15. Miyabayashi, K., Naito, Y., Miyake, M., Tsujimoto, K.: Quantitative capability of electrospray ionization Fourier transform ion cyclotron resonance mass spectrometry for a complex mixture. Eur. J. Mass Spectrom. 6, 251-258 (2000) 
16. Hughey, C.A., Minardi, C.S., Galasso-Roth, S.A., Paspalof, G.B., Mapolelo, M.M., Rodgers, R.P., Marshall, A.G., Ruderman, D.L.: Naphthenic acids as indicators of crude oil biodegradation in soil, based on semiquantitative electrospray ionization Fourier transform ion cyclotron resonance mass spectrometry. Rapid Commun. Mass Spectrom. 22, 3968-3976 (2008)

17. Mapolelo, M.M., Rodgers, R.P., Yen, A.T., Debord, J.D., Asomaning, S., Marshall, A.G.: Electrospray ionization FT-ICR mass spectrometry of "ARN" naphthenic acids in sodium and calcium naphthenate deposits, and crudes: Extraction and quantification. 235th ACS Meeting, New Orleans, LA (2008)

18. Purcell, J.M., Juyal, P., Kim, D.-G., Rodgers, R.P., Hendrickson, C.L., Marshall, A.G.: Sulfur speciation in petroleum: Atmospheric pressure photoionization or chemical derivatization and electrospray ionization Fourier transform ion cyclotron resonance mass spectrometry. Energy Fuels 21, 2869-2874 (2007)

19. Purcell, J.M., Hendrickson, C.L., Rodgers, R.P., Marshall, A.G.: Atmospheric pressure photoionization Fourier transform ion cyclotron resonance mass spectrometry for complex mixture analysis. Anal. Chem. 78, 5906-5912 (2006)

20. Al-Hajji, A.A., Muller, H., Koseoglu, O.R.: Characterization of nitrogen and sulfur compounds in hydrocracking feedstocks by Fourier transform ion cyclotron mass spectrometry. Oil Gas Sci. Technol. 63, 115-128 (2008)

21. Smith, D.R., Robb, D.B., Blades, M.W.: Comparison of dopants for charge exchange ionization of nonpolar polycyclic aromatic hydrocarbons with reversed-phase LC-APPI-MS. J. Am. Soc. Mass Spectrom. 20, 73-79 (2009)

22. Kauppila, T.J., Bruins, A.P., Kostiainen, R.: Effect of the solvent flow rate on the ionization efficiency in atmospheric pressure photoionization-mass spectrometry. J. Am. Soc. Mass Spectrom. 16, 1399-1407 (2005)
23. Purcell, J.M., Hendrickson, C.L., Rodgers, R.P., Marshall, A.G.: Atmospheric pressure photoionization proton transfer for complex organic mixtures investigated by Fourier transform ion cyclotron resonance mass spectrometry. J. Am. Soc. Mass Spectrom. 18, 1682 1689 (2007)

24. Hughey, C.A., Hendrickson, C.L., Rodgers, R.P., Marshall, A.G.: Elemental composition analysis of processed and unprocessed diesel fuel by electrospray ionization Fourier transform ion cyclotron resonance mass spectrometry. Energy Fuels 15, 11861193 (2001)

25. Ruiz-Guerrero, R., Vendeuvre, C., Thiebaut, D., Bertoncini, F., Espinat, D.: Comparison of comprehensive two-dimensional gas chromatography coupled with sulfur-chemiluminescence detector to standard methods for speciation of sulfur-containing compounds in middle distillates. J. Chromatogr. Sci. 44, 566-573 (2006)

26. Choudhary, T.V., Malandra, J., Green, J., Parrott, S., Johnson, B.: Towards clean fuels: molecular-level sulfur reactivity in heavy oils. Angew. Chem. Int. Ed. 45, 3299-3303 (2006)

27. Muller, H., Al-Jawad, H.H., Adam, F., Al-Hajji, A.A.: Quantitative Analysis of Aromatic compounds in petroleum by FT-ICR-MS. 59th ASMS Conference, Denver, CO (2011)

28. Schoenmakers, P.J., Oomen, J.L.M.M., Blomberg, J., Genuit, W., Van Velzen, G.: Comparison of comprehensive two-dimensional gas chromatography and gas chromatography-mass spectrometry for the characterization of complex hydrocarbon mixtures. J. Chromatogr. A 892, 29-46 (2000)

29. Muller, H., Adam, F., Al-Hajji, A.A., Koseoglu, O.R.: Comparison of FT-ICR MS and comprehensive gas chromatography for the analysis of petroleum refining streams: How to get quantitative FT-ICR MS data? 58th ASMS Conference, Salt Lake City, UT (2010) 